\title{
Correlation of the refractive index change at the surface and inside phosphate glass upon femtosecond laser irradiation
}

\author{
Daniel Puerto, ${ }^{1,2,3}$ Jan Siegel, ${ }^{1, *}$ Andres Ferrer, ${ }^{1}$ Javier Hernandez-Rueda, ${ }^{1}$ and Javier Solis ${ }^{1}$ \\ ${ }^{1}$ Laser Processing Group, Instituto de Optica - CSIC, Madrid, Spain \\ ${ }^{2}$ Nanophotonics Technology Center, Universitat Politècnica de València, Valencia, Spain \\ ${ }^{3} e$-mail: danielpuerto@ntc.upv.es \\ ${ }^{*}$ Corresponding author: j.siegel@io.cfmac.csic.es
}

Received May 31, 2012; revised July 26, 2012; accepted July 27, 2012;

posted July 27, 2012 (Doc. ID 169610); published September 6, 2012

\begin{abstract}
A study of the surface modification induced by single femtosecond laser pulses in phosphate glass has revealed surface swelling at fluences just below the ablation threshold. This behavior is different from that observed in other dielectric materials. Optical micrographs obtained with monochromatic light show a pattern of Newton rings within the swollen region whose number scales inversely with the illumination wavelength, acting as a micro Fabry-Perot etalon. The swollen surface layer has lower refractive index than the bulk glass and can reach a maximum thickness of $820 \mathrm{~nm}$. We relate these findings to results obtained during subsurface waveguide writing inside phosphate glass, which also show a refractive index decrease at energies near threshold for waveguide fabrication. We have identified low density free-electron plasma to be the trigger of the refractive index change. (C) 2012 Optical Society of America
\end{abstract}

OCIS codes: $\quad 320.2250,320.7130,140.3390,350.5400$.

\section{INTRODUCTION}

The increasing demands of optical communications over the past decades have stimulated the fabrication of compact, waveguide integrated optical devices [1] . In this context, the use of femtosecond (fs) laser-based fabrication techniques has demonstrated an immense potential and versatility over more conventional photonic device fabrication techniques [2-4]. When an ultrafast laser beam is focused inside a dielectric material, the characteristic properties of fs laser pulses (short pulse duration and high peak irradiance) enable a controlled energy deposition via nonlinear absorption in a region limited to the focal volume with submicrometric resolution [5]. This way, structural and/or refractive index modifications can be produced inside glasses and crystals, which allows direct writing of optical waveguides and more sophisticated active and passive photonic devices [2,3,5].

Despite these achievements, little is known about the relation between material changes occurring upon fs laser surface processing and those taking place upon subsurface processing. The reason is that, unlike subsurface (bulk) processing, surface processing is mostly performed at energies above the ablation threshold [6]. An exception is found for fused silica, for which a relation could be established between laserinduced densification in the bulk and at the surface at energies below the ablation threshold, both triggered by transient freeelectron plasmas [ㄱ-9]. However, such studies are missing for technologically more relevant materials for the fabrication of active photonic devices, such as phosphate glasses $[\underline{1}, \underline{3}, \underline{5}, \underline{10}, \underline{11}]$. Recent studies have contributed to unravel the structural mechanisms responsible for fs laser-induced refractive index changes upon subsurface processing in these glasses $[\underline{12}, \underline{13}, \underline{14}]$.
In this paper we investigate the modification of a phosphate glass upon surface processing at laser fluences near the ablation threshold and relate the results obtained to those observed during bulk processing. In addition, we identify experimental conditions to produce at the surface a micro Fabry-Perot etalon with low finesse.

\section{EXPERIMENTAL}

The material under study was a commercial phosphate glass doped with 2 wt. \% $\mathrm{Er}^{3+}$ and 2.5 wt. $\% \mathrm{Yb}^{3+}$ ("MM2" by Kigre), which has a bandgap of $E_{g}=3.6 \mathrm{eV}$. The laser system used for irradiation was a commercial amplified fs laser system (100 fs, $1 \mathrm{kHz}$ repetition rate). Surface irradiation was performed by exposing the sample to a single $s$-polarized pulse, focused at the surface with a $150 \mathrm{~mm}$ lens at an angle of $54^{\circ}$, producing an elliptical spot with a Gaussian intensity distribution $\left(110 \mu \mathrm{m} \times 70 \mu \mathrm{m}, 1 / e^{2}\right.$ diameter, determined using the method proposed by Liu [15]). A fs optical microscope was installed in situ to record transient reflectivity images of the irradiated region by illuminating it with a frequencydoubled fs probe pulse at $400 \mathrm{~nm}$, delayed with respect to the pump pulse. "We have chosen the elevated angle of incidence in order to avoid that the reflection of the pump laser light by the sample propagates towards the CCD camera and might not be fully filtered by the interference filter placed before." Details of the experimental setup can be found elsewhere [7,8]. Ex situ analysis of optical and topographic changes in the processed surface has been performed with an optical microscope $(\mathrm{OM})$ and optical interferometric microscope (OIM), respectively.

For subsurface processing the pulses are focused inside the sample with a microscope objective (NA 0.26). In order to 
minimize nonlinear propagation effects inside the sample before reaching the focal region, the pulses are stretched to $260 \mathrm{fs}$ and circularly polarized [9]. Moreover, in order to generate a disklike focal region and thus circular cross section of the written waveguide, the circular laser beam has been spatially shaped by passing a $350 \mu \mathrm{m}$ wide horizontal slit before entering the microscope objective $[5,9,11]$. During irradiation at $1 \mathrm{kHz}$ pulse repetition rate, the sample is translated at $100 \mu \mathrm{m} / \mathrm{s}$ perpendicular to the incident beam axis, parallel to the slit's long axis, while imaging plasma emission produced during processing with a laterally installed in situ optical microscope [11]. Ex situ characterization of the written waveguides was performed using an $\mathrm{OM}$ in trans-illumination. Characterization of local changes in the material density is induced by laser irradiation performed by confocal microfluorescence imaging, employing $488 \mathrm{~nm}$ excitation. In particular, the spectral shift of the $553 \mathrm{~nm} \mathrm{Er}^{+}$emission line has been analyzed, which is indicative of matrix dilatation or densification [14].

\section{RESULTS AND DISCUSSION}

Figure 1(a) shows several topography sections through the center of surface regions exposed to single fs laser pulses at fluences above and below the ablation threshold (defined as the onset of material removal). At the highest fluence used $\left(4.4 \mathrm{~J} / \mathrm{cm}^{2}\right)$ the maximum crater depth is $\approx 140 \mathrm{~nm}$, displaying relatively steep walls, surrounded by an annular protrusion of up to $40 \mathrm{~nm}$ height. This protrusion could in principle be associated to resolidified molten material ejected toward the crater edge by the recoil pressure of the ablating material acting upon the molten layer underneath [16]. However, a detailed look at the topography evolution at lower fluences rules out this mechanism. At $3.6 \mathrm{~J} / \mathrm{cm}^{2}$, the crater diameter and depth have strongly decreased while the width of protrusion has increased. At $3.5 \mathrm{~J} / \mathrm{cm}^{2}$, no crater is observed but the protrusion is still pronounced, displaying a further increased width. These results demonstrate unambiguously that the protrusion in this glass is a direct consequence of laser irradiation
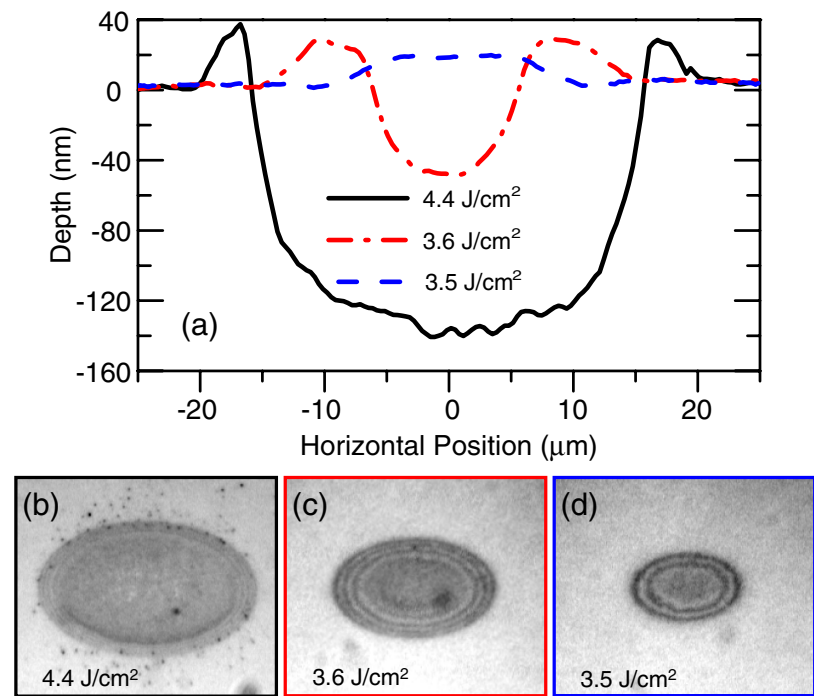

Fig. 1. (Color online) (a) Topography profiles along the long axis of single pulse irradiated regions in phosphate glass at three different laser fluences (see labels). (b)-(d) Optical micrographs of the laser-exposed regions shown in (a). The field of view is in all cases $46 \times 39 \mu \mathrm{m}^{2}$. below the ablation threshold, leading to surface swelling, and not a by-product of ablation. The threshold value found for surface swelling is $3.25 \mathrm{~J} / \mathrm{cm}^{2}$, compared to an ablation threshold of $3.58 \mathrm{~J} / \mathrm{cm}^{2}$.

We have inspected the irradiated regions with an optical microscope in reflection. The corresponding micrographs displayed in Figs. 1(b)-1(d) show an overall darkening together with an elliptic ring pattern, which is spatially coincident with the region of the protrusion. The ring pattern is reminiscent of Newton rings, an interference pattern caused by the reflection of light between two surfaces: a spherical surface and an adjacent flat surface. We have confirmed this hypothesis by performing optical microscopy using two monochromatic illumination sources with different wavelength. Figure 2(a) shows a micrograph of a region irradiated at $3.9 \mathrm{~J} / \mathrm{cm}^{2}$ illuminated at $460 \mathrm{~nm}$ wavelength, nicely featuring the ring pattern. Figure 2(b) shows the very same region and field of view, illuminated at $800 \mathrm{~nm}$. A comparison of both images shows that for $800 \mathrm{~nm}$ light, the number of fringes (3) is approximately half the one observed for $460 \mathrm{~nm}$ light (5). The product of wavelength and fringe number is constant within the error of this first estimation, confirming that the rings are caused by an optical interference phenomenon. This can also be seen in the corresponding horizontal reflectivity profiles [Figs. 2(c) and $\underline{2(\mathrm{~d})}$ ], which reveal a maximum modulation depth $\Delta R=$ -0.07 for $460 \mathrm{~nm}$ light and $\Delta R=-0.09$ for $800 \mathrm{~nm}$ light. This structure behaves like a micro Fabry-Perot etalon with a low finesse.

The relative refractive index change $\Delta n / n$ responsible for the appearance of the Newton rings can be calculated assuming a two-layer system. The lower layer is assumed infinite and its refractive index $n$ corresponds to that of bulk phosphate glass at a given wavelength. The thickness $d$ and refractive index $n_{1}$ of the upper layer is varied in the simulation until a match with the number of fringe oscillations and their modulation depth observed is obtained. The results are shown in Figs. 2(e) and 2(f) for $460 \mathrm{~nm}$ and $800 \mathrm{~nm}$ illumination,
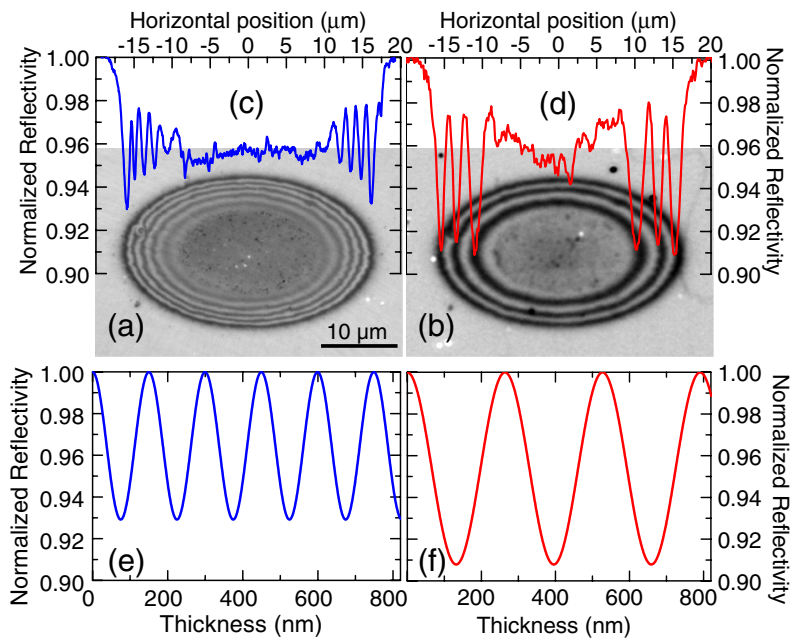

Fig. 2. (Color online) Two optical micrographs show the same region, irradiated by a single pulse at $3.9 \mathrm{~J} / \mathrm{cm}^{2}$, illuminated with $460 \mathrm{~nm}$ (a) and $800 \mathrm{~nm}$ (b). The field of view is in both cases $40 \times 27 \mu \mathrm{m}^{2}$. On top, the corresponding normalized reflectivity profiles are shown (c), (d). The lower graphs show the calculated reflectivity modulation for a system with a top layer of reduced refractive index as a function of its thickness for $460 \mathrm{~nm}$ (e) and $800 \mathrm{~nm}$ light (f), using $\Delta n / n_{460 \mathrm{~nm}} \approx-0.008$ and $\Delta n / n_{800 \mathrm{~nm}} \approx-0.011$, respectively. 
respectively. Consistently, both calculations yield a thickness $d=820 \pm 20 \mathrm{~nm}$ for the upper layer to match the number of experimentally observed rings $(=5$ for $460 \mathrm{~nm}$ and $=3$ for $800 \mathrm{~nm}$ ). The refractive index changes obtained from the calculations are $\Delta n / n_{460 \mathrm{~nm}}=-0.008 \pm 0.0005$ and $\Delta n / n_{800 \mathrm{~nm}}=-0.011 \pm 0.0003$. The value obtained for $460 \mathrm{~nm}$ is likely underestimated due to the slight absorption in the material at this wavelength.

We have investigated the origin of surface swelling and refractive index decrease by means of imaging the transient free-electron plasma formed upon irradiation using a fs pump-probe microscope [7,8]. The delay of probe pulse was chosen to be 1 ps since in this delay range the plasma features its maximum transient reflectivity [8]. The image recorded is shown in Fig. 3(a), showing a weak transient reflectivity increase in the very region, which after irradiation displays Newton rings [Fig. 3(b)] and surface swelling [Fig. 3(c)]. Using a simple Drude model, an estimation of the plasma density can be obtained [8], yielding a value of $n_{e}=1.1 \times 10^{22} \mathrm{~cm}^{-3}$. The presence of Newton rings in irradiated phosphate glass implies a sharp interface, which suggests that the plasma relaxes and transfers energy to the lattice, thereby melting the material. The sharp interface is formed upon rapid solidification into a less dense structure, which indicates a normal glass transition [17,18]. A different mechanism takes place in fused silica at similar plasma densities $\left(n_{e}=1.4 \times 10^{22} \mathrm{~cm}^{-3}\right)$, where surface depression and lattice densification is observed [8]. This behavior is generally attributed to the anomalous glass transition in fused silica, which increases the material density upon rapid solidification $[17,18]$. Yet the absence of Newton rings in the densified region in fused silica indicates the absence of a sharp interface, therefore questioning the presence of melting and opening the possibility of plasma-induced densification [8]. The absence of melting in fused silica under these conditions is also

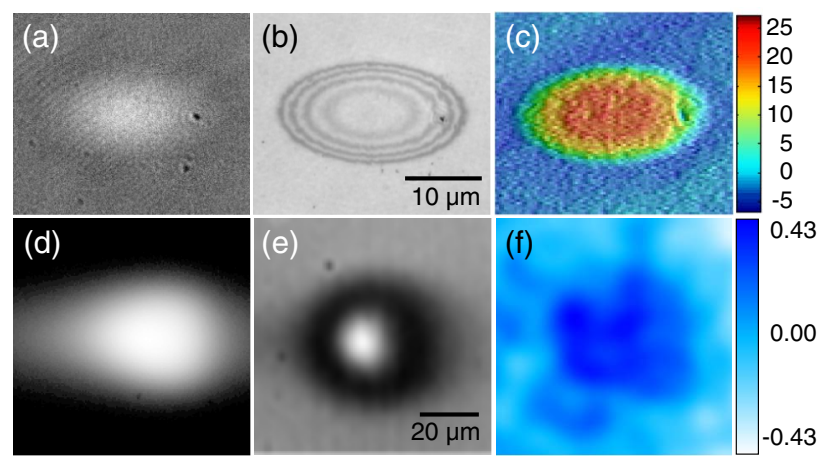

Fig. 3. (Color online) Comparison of results obtained in phosphate glass upon surface irradiation (top row) and bulk irradiation (bottom row). Top row (field of view $31 \times 26 \mu \mathrm{m}^{2}$ ): Single pulse irradiation at $3.55 \mathrm{~J} / \mathrm{cm}^{2}$. (a) Transient free-electron plasma at a delay of $1 \mathrm{ps}$ as measured by fs microscopy (see text). (b) Appearance as measured by optical microscopy using $460 \mathrm{~nm}$ light, featuring Newton rings. (c) Topography map in false color with units given in $\mathrm{nm}$. Bottom row (field of view $80 \times 80 \mu \mathrm{m}^{2}$ ): Irradiation at $1 \mathrm{kHz}$ and sample scanning at $100 \mu \mathrm{m} / \mathrm{s}, 16.0 \mathrm{~J} / \mathrm{cm}^{2}$. The laser is incident from the left. (d) Time-integrated free electron plasma as measured by plasma emission microscopy (in logarithmic intensity scale). (e) Appearance as measured by microscopy, featuring a lowered transmission. (f) Spectral shift of the $\mathrm{Er}^{+}$emission line at $533 \mathrm{~nm}$ as measured by confocal microfluorescence imaging (taken and adapted from [16]). The units of the colour bar are $\mathrm{cm}^{-1}$. supported by the much higher glass transition temperature $\left(T_{m}=1600{ }^{\circ} \mathrm{C}\right)$ compared to phosphate glass $\left(T_{m}=552{ }^{\circ} \mathrm{C}\right)$. The measured electron densities can be related to the critical electron density for optical breakdown, which is given by $n_{c}=\varepsilon_{0} m_{e} \omega^{2} / e^{2}$, with $\omega$ being the probe laser frequency, $m_{e}$ and $e$ the electron mass and charge, respectively, and $\varepsilon_{0}$ the dielectric constant. This yields a value of $n_{c}=$ $7 \times 10^{21} \mathrm{~cm}^{-3}$ for our conditions and implies that the mechanisms of surface swelling/lattice dilation in phosphate glass and surface depression/lattice densification in fused silica occur close above the breakdown threshold.

We have also investigated the relation between surface swelling and bulk modification generated upon subsurface waveguide writing. The origin of the refractive index change induced in the bulk has been investigated by several groups [12-14], and has been shown to strongly depend on the thermal effects appearing at high rep. rates. (>approx. $100 \mathrm{kHz}$ ) [19]. In order to compare with the former single-pulse surface experiments, we have tried to establish a relation only to waveguides written in the low repetition rate regime $(1 \mathrm{kHz})$, in which the modification of the material is a consequence of the effect of each individual pulse [19]. We have written a waveguide, using the experimental conditions described in the experimental section, by focusing the laser at normal incidence at a depth of $1.56 \mathrm{~mm}$ inside the sample and translating the sample during the irradiation. Figure $3(\mathrm{~d})$ shows a side view of the free-electron plasma produced during writing inside phosphate glass, displayed in logarithmic intensity scale in order to reveal the weaker off-center regions. At the pulse energy used ( $4 \mu \mathrm{J}, 16 \mathrm{~J} / \mathrm{cm}^{2}$ peak fluence), the written structure is characterized by an overall reduced light transmission (except the very center), as shown in the transmission microscopy image in Fig. 3(e).

After writing, we have investigated the structural changes induced in this waveguide by confocal microfluorescence imaging. Figure 3(f) shows a map of the spectral shift of the $553 \mathrm{~nm} \mathrm{\textrm {Er } ^ { 3 + }}$ emission line of the region containing the waveguide upon excitation at $488 \mathrm{~nm}$, featuring a significant blue shift. As shown in [14], a blue shift is accompanied by an increase of the emission intensity of this emission line, a decrease of the emission intensity of the $\mathrm{Yb}^{3+}$ emission at $976 \mathrm{~nm}$, and an increase of the upconversion efficiency upon excitation at $980 \mathrm{~nm}$. All these features are consistent with matrix dilatation and a refractive index decrease. The limited contribution of other possible mechanisms (such as a change in local polarizability) to the refractive index change cannot be ruled out, although their typical manifestation (overall fluorescence quenching) has not been observed. In this sense, the blueshift as observed in Fig. 3(f) can be interpreted as a direct signature of matrix dilatation and refractive index decrease. This behavior of laser-induced modifications inside phosphate glass is consistent with the one observed at the surface; swelling and refractive index decrease as shown in Figs. 3(b) and 3(c). It implies that comparable material changes are induced at the surface and inside the material, despite the different processing conditions (single pulse versus multiple pulses, different local fluences) and the different plasma densities involved (20 times less inside bulk as measured in [9]). We attribute this result to the negligible thermal accumulation effects in the case of multiple pulse irradiation due to the low pulse repetition rate used. 


\section{CONCLUSIONS}

We have shown that single pulse fs laser irradiation of a phosphate glass surface leads to surface swelling within a narrow fluence range. This structure acts as a micro Fabry-Perot etalon and consists of a laser-produced top layer of lower refractive index than the bulk material, separated by a sharp optical interface. The maximum layer thickness has been determined to $d=820 \mathrm{~nm}$ with a maximum refractive index change of $\Delta n / n=-0.011$. As the origin of the modification, a laser-induced, transient free electron plasma with a peak density of $1.1 \times 10^{22} \mathrm{~cm}^{-3}$ was identified. We could relate these results to those obtained during subsurface waveguide writing at $1 \mathrm{kHz}$ repetition rate, also generating a refractive index decrease. Despite the different conditions in terms of number of pulses and plasma densities involved, the material response is similar at the surface and inside bulk under the processing conditions used, which we attribute to the low pulse repetition rate used, thus minimizing thermal accumulation effects. The simple fabrication process of a surface layer with different refractive index and sharp optical interface by a single laser pulse could find numerous applications in telecommunication, including micro Fabry-Perot etalons.

\section{ACKNOWLEDGMENTS}

We thank Daniel Jaque from the Fluorescence Imaging Group at the Universidad Autónoma de Madrid for providing access to and helping with the confocal microfluorescence imaging system, as well as for many fruitful discussions. We are also grateful to Susana Marcos and Carlos Dorronsoro from IO-CSIC for providing access and assistance to the OIM. This work has been partially supported by the Spanish TEC 200801183 and TEC2011-22422. D. Puerto acknowledges his grant of the Spanish Ministry of Science and Education under TEC 2005-0007 and JCI-2010-07479.

\section{REFERENCES}

1. J. D. B. Bradley and M. Pollnau, "Erbium-doped integrated waveguide amplifiers and lasers," Laser Photon. Rev. 5, 368-403 (2011).

2. K. Hirao, T. Mitsuyu, J. Si, and J. Qiu, Active Glass for Photonic Applications: Photoinduced Structures and Their Application (Springer, 2001).

3. G. Della Valle, R. Osellame, and P. Laporta, "Micromachining of photonic devices by femtosecond laser pulses (Review article)," J. Opt. A Pure Appl. Opt. 11, 013001 (2009).

4. L. Sansoni, F. Sciarrino, G. Vallone, P. Mataloni, A. Crespi, R. Ramponi, and R. Osellame, "Polarization entangled state measurement on a chip," Phys. Rev. Lett. 105, 1-4 (2010).

5. R. Osellame, G. Cerullo, and R. Ramponi, Femtosecond Laser Micromaching: Photonic and Microfluidic Devices in Trans- parent Materials, Vol. 123 of Topics in Applied Physics (Springer, 2012).

6. A. Ostendorf, F. Korte, G. Kamlage, U. Klug, J. Koch, J. Serbin, N. Baersch, T. Bauer, and B. N. Chichkov, "Applications of femtosecond lasers in 3D micromachining," in 3D Laser Microfabrication Principles and Applications, H. Misawa and S. Juodkazis, eds. (Wiley-VCH, 2006).

7. J. Siegel, D. Puerto, W. Gawelda, G. Bachelier, J. Solis, L. Ehrentraut, and J. Bonse, "Plasma formation and structural modification below the visible ablation threshold in fused silica upon femtosecond laser irradiation," Appl. Phys. Lett. 91, 082902 (2007).

8. D. Puerto, J. Siegel, W. Gawelda, M. Galvan-Sosa, L. Ehrentraut, J. Bonse, and Y. J. Solis, "Dynamics of plasma formation, relaxation, and topography modification induced by femtosecond laser pulses in crystalline and amorphous dielectrics," J. Opt. Soc. Am. B 27, 1065-1076 (2010).

9. W. Gawelda, D. Puerto, J. Siegel, A. Ferrer, A. Ruiz de la Cruz, H. Fernández, and J. Solis, "Ultrafast imaging of transient electronic plasmas produced in conditions of femtosecond waveguide writing in dielectrics," Appl. Phys. Lett. 93, 121109 (2008).

10. F. Patel, S. DiCarolis, and P. Lum, "A compact high-performance optical waveguide amplifier," IEEE Photon. Technol. Lett. 16, 2607-2609 (2004).

11. A. Ferrer, A. Ruiz de la Cruz, D. Puerto, W. Gawelda, J. A. Vallés, M. A. Rebolledo, V. Berdejo, J. Siegel, and J. Solis, "In situ assessment and minimization of nonlinear propagation effects for femtosecond-laser waveguide writing in dielectrics," J. Opt. Soc. Am. B 27, 1688-1692 (2010).

12. L. B. Fletcher, J. J. Witcher, W. B. Reichman, A. Arai, J. Bovatsek, and D. M. Krol, "Changes to the network structure of Er-Yb doped phosphate glass induced by femtosecond laser pulses," J. Appl. Phys. 106, 083107 (2009).

13. D. J. Little, M. Ams, P. Dekker, G. D. Marshall, and M. J. Withford, "Mechanism of femtosecond-laser induced refractive index change in phosphate glass under a low repetition-rate regime," J. Appl. Phys. 108, 033110 (2010).

14. A. Ferrer, D. Jaque, J. Siegel, A. Ruiz de la Cruz, and J. Solis, "Origin of the refractive index modification of femtosecond laser processed doped phosphate glass," J. Appl. Phys. 109, 093107 (2011).

15. J. M. Liu, "Simple technique for measurements of pulsed Gaussian-beam spot sizes," Opt. Lett. 7, 196 (1982).

16. A. Ben-Yakar, A. Harkin, J. Ashmore, R. L. Byer, and H. A. Stone, "Thermal and fluid processes of a thin melt zone during femtosecond laser ablation of glass: the formation of rims by single laser pulses," J. Phys. D 40, 1447 (2007).

17. R. Brückner, "Properties and structure of vitreous silica I," J. Non-Cryst. Solids 5, 123-175 (1970).

18. L. Bressel, D. de Ligny, C. Sonneville, V. Martinez, V. Mizeikis, R. Buividas, and S. Juodkazis, "Femtosecond laser induced density changes in $\mathrm{GeO} 2$ and $\mathrm{SiO} 2$ glasses: fictive temperature effect," Opt. Mater. Express 1, 605-613 (2011).

19. S. Eaton, H. Zhang, P. Herman, F. Yoshino, L. Shah, J. Bovatsek, and A. Arai, "Heat accumulation effects in femtosecond laserwritten waveguides with variable repetition rate," Opt. Express 13, 4708-4716 (2005). 